\title{
Localized and Delocalized Electronic Excitations in Biological and Artificial Antenna Complexes
}

\author{
Sergei Tretiak ${ }^{1}$, Cris Middleton ${ }^{2}$, Vladimir Chernyak ${ }^{2}$, and Shaul Mukamel ${ }^{2}$.
}

(1) Theoretical Division, Los Alamos National Laboratory, Mail stop B262, Los Alamos, NM 87545, fax: (505) 665-4063, serg@cnls.lanl.gov,

(2) Chemistry Department, University of Rochester, Rochester, NY

\begin{abstract}
The Collective Electronic Oscillators (CEO) approach based on the Time-Dependent Hartree-Fock approximation is applied to analyze localization and delocalization of electronic excitations, charge and energy transfer in LH2 aggregates (biological antenna complexes) and in phenylacetylene dendrimers (artificial antenna macromolecules). Two-dimensional analysis of transition density matrices indicates that electron-hole pairs created upon optical excitation in LH2 aggregate are localized within separate chromophores. In addition LH2 has a band of low-lying charge-transfer states unaccessible with linear absorption spectroscopy. In phenylacetylene dendrimers, electronic excitation are shown to be localized within linear segments connected by benzene rings substituted at the meta- position. These results are used to construct effective Frenkel exciton hamiltonians in these biological and artificial light-harvesting antenna complexes.
\end{abstract}

\section{Introduction}

Advances in organic synthesis yield supermolecules with precisely defined structures. These include molecular rods and wires ${ }^{1}$, dendrimeric nanostructures ${ }^{2}$, higher fullerenes and metallofullerenes ${ }^{3}$, giant cage-like receptors ${ }^{4}$, and organic superlattices ${ }^{5}$. In addition, molecular aggregates are common in biological processes such as light-harvesting complexes in photosynthesis ${ }^{6}$. X-ray and NMR techniques provide the geometry of these structures which consist of thousands of atoms ${ }^{7}$.

Optical spectroscopy is an important tool in the study of photophysics and photochemistry ${ }^{8}$, charge and energy transfer ${ }^{9}$, intermolecular interactions and bonding in these systems. The theoretical investigation is complicated by the delocalized nature of electronic excitations, strong electron correlations, and vibronic coupling. An important challenging goal is to relate the electronic states and spectra of aggregates to those of their basic building blocks - the monomers. By doing so it should become possible to get a better microscopic insight into the nature of their electronic excitations and to predict qualitative features of complex large systems using simple, readily available information. The problem is simplified considerably for molecular aggregates made of well separated chromophores, whose interactions are purely Coulombic. Electron exchange is then negligible, each chromophore retains its own electrons, and the system may be described using the Frenkel exciton Hamiltonian ${ }^{10,11}$. The situation is much more complex when electronic states are delocalized among the chromophores. No obvious perturbative theory exists in this case. The calculations of optical excitations are tedious and provide no simple rules of thumb for predicting spectroscopic trends.

In this paper we apply the collective electronic oscillator (CEO) approach ${ }^{12,13}$ to study the electronic excitations of Bchl-a aggregates of LH2 complex of Rs. molischianum and dendrimers made up of phenylacetylene repeat unit in a self-similar fashion around the core. The CEO method provides an effective computational scheme for electronic excitations of large molecules. The results allow a direct real-space interpretation of the spectra, illustrating the role of geometric confinement and exciton localization. Our calculations are used to construct effective Frenkel exciton hamiltonian in the LH2 complex and dendrimers and analyze the photoexcitation energy transfer within the aggregate.

\section{Computational method}

The numerical CEO-INDO/S procedure for calculating electronic structure has been described in detail elsewhere ${ }^{12,13}$. The ZINDO code was first applied to generate the INDO/S hamiltonian using geometries obtained from crystal structures of LH2 complex of Rs. molischianum and optimization of dendrimer structures at the AM1 level. We next calculated the Hartree-Fock ground-state density matrices which are the input to the following CEO calculation. The CEO procedure ${ }^{12,13}$ was finally applied to compute the 
linear-absorption spectra and the relevant transition density matrices (denoted the electronic normal modes $\xi_{\mathrm{v}}$ ), which connect the optical response with the underlying electronic motions. Each mode is a matrix representing the electronic transition between the ground state $|g\rangle$ and an electronically excited state $|v\rangle$ : $\xi_{\mathrm{v}}=\left\langle\mathrm{v}\left|\mathrm{c}_{\mathrm{m}}{ }^{+} \mathrm{c}_{\mathrm{n}}\right| \mathrm{g}\right\rangle$, where $\mathrm{c}_{\mathrm{m}}{ }^{+}\left(\mathrm{c}_{\mathrm{m}}\right)$ are creation (annihilation) operators of an electron at the m'th atomic orbital. The modes can be computed as eigenmodes of the linearized time-dependent Hartree-Fock (TDHF) equations of motion for the density matrix driven by the external field, totally avoiding the explicit calculation of many-electron excited-state wavefunctions. The eigenfrequencies $\Omega_{v}$ of these equations provide the optical transition frequencies ${ }^{12,13}$. The numerical effort involved in computing these eigenvalues and eigenvectors is greatly reduced by using the oblique Lanczos algorithm. Transition dipole moments $\mu_{v}=\operatorname{Tr}\left(\mu \xi_{v}\right)$ were then calculated using the dipole moment operator $\mu=\Sigma_{\mathrm{nm}} \mu_{\mathrm{mn}} \mathrm{c}_{\mathrm{m}}{ }^{+} \mathrm{c}_{\mathrm{n}}$, and $\mathrm{f}_{v}=2 \Omega_{v} \mu_{v}{ }^{2}$ is the oscillator strength of the $g$ to $v$ transition.

The electronic modes represent collective motions of electrons and holes. They carry substantially less information than the many-electron eigenstates but more than required for calculating molecular polarizabilities and spectroscopic observables. The diagonal elements $\left(\xi_{\mathrm{v}}\right)_{\mathrm{nn}}$ represent the net charge induced on the n'th atomic orbital by an external field, whereas $\left(\xi_{\mathrm{v}}\right)_{\mathrm{nm}} \mathrm{n} \neq \mathrm{m}$ is the dynamical bond-order (or electronic coherence) representing the joint amplitude of finding an electron on orbital $\mathrm{m}$ and a hole on orbital $n$.

Figure 1. Graphical representation of the CEO calculated excitonic couplings (in $\mathrm{cm}^{-1}$ ) of the LH2 complex.
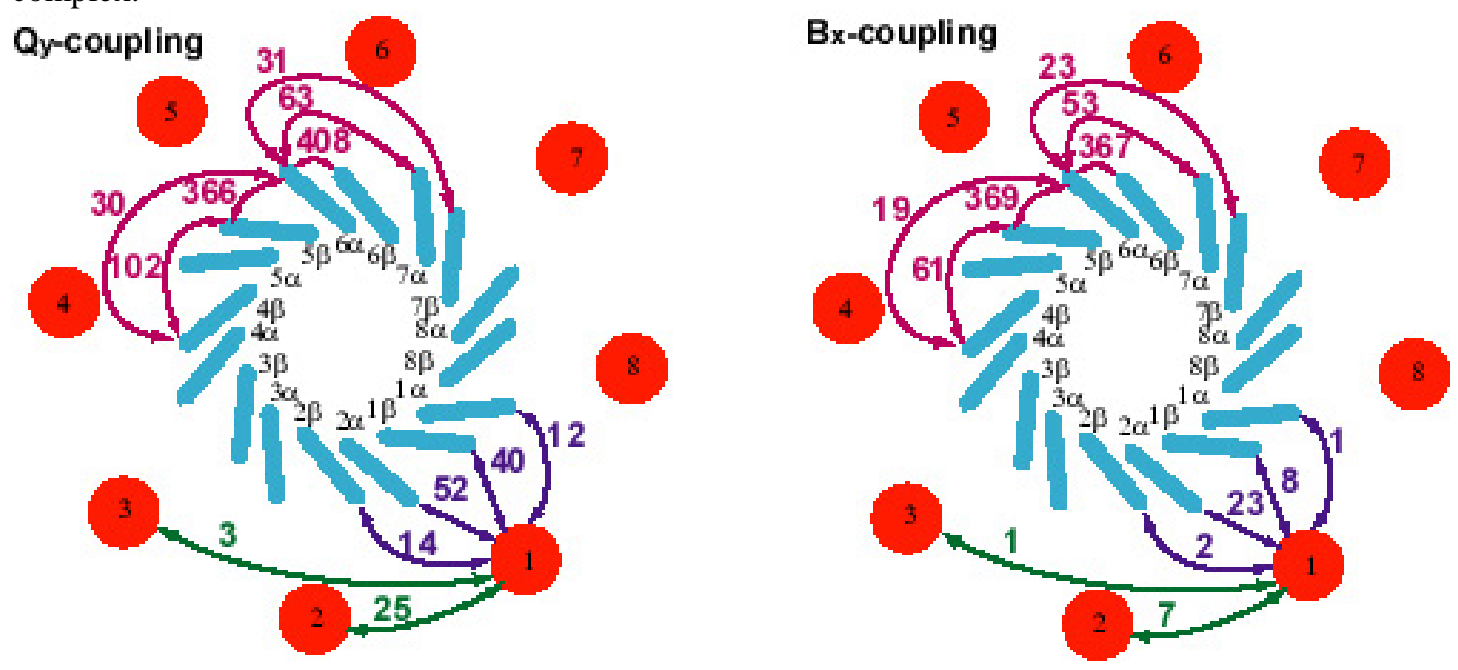

Frenkel exciton hamiltonian for $\mathrm{LH} 2$ photosynthetic antenna

The primary processes of photosynthesis, the capture of sunlight, and the subsequent conversion into chemical energy, constitute the very basis of all life. ${ }^{6}$ The photosynthetic unit (PSU) of purple bacteria is the most studied and the best characterized among the known photosynthetic systems. ${ }^{14,15}$ The $2.5 \mathrm{~A}$ resolution geometrical structure of the Rs. molischianum LH2 complex ${ }^{7}$ shows 24 bacteriochlorophyll-a (BChl-a) monomers arranged in two rings: 8 weakly coupled BChla molecules form the outer ring which is responsible for the higher energetic $\mathrm{B} 800$ band, while 16 strongly interacting $\mathrm{BChl}$-a of the inner ring form the lower energetic B850 band (see Fig.1). Energy-migration in the antenna complexes depends on electronic coupling between the donor and acceptor chromophores. A point dipole approximation (PDA) is generally applied to compute this electrostatic interaction. The PDA assumes that the chromophore sizes are small compared to their separation and the coupling is then given by the interaction between donor and acceptor transition dipole moments. The PDA which is routinely used for evaluating coupling constants does not hold for calculations of J between closely lying chromophores such as in the LH2 system ${ }^{16,17}$ since the chromophore sizes are not small compare to their separation.

To explore intermolecular interactions we consider electronic spectra of dimers composed from two chromophores (monomers). Panel A of Fig. 2 displays the $\alpha \beta B 850$ heterodimer. Computed linear absorption spectrum (panel B) shows that each monomeric transition has a Davydov splitting ${ }^{10}$ in the dimer 
spectra. To trace the origin of the various peaks we had examined the corresponding electronic modes. Two-dimensional plots of these matrices shows hole and electron dynamics along the $\mathrm{x}$ and $\mathrm{y}$ axes, respectively. Atom labeling runs over $\beta$ B850 first then over $\alpha$ B850, following the pattern of Fig. 2A. Panel $\rho$ shows the ground state density matrix of $\alpha \beta B 850$. As expected, it is simply the superposition of the monomeric ground states and both Bchls-a are clearly identified. Panels $\mathrm{Q}_{\mathrm{y} 1}$ and $\mathrm{Q}_{\mathrm{y} 2}$ show a pair of states corresponding to the $\mathrm{Q}_{\mathrm{y}}$ mode. The corners of the plot represent the monomers. They are completely uncoupled, i.e. there is no off-diagonal coherences between monomers. $\mathrm{Q}_{\mathrm{y} 1}$ and $\mathrm{Q}_{\mathrm{y} 2}$ are therefore symmetric and antisymmetric combinations of the monomeric excited states wavefunctions. The interaction between monomers is purely electrostatic, which establishes the applicability of the Frenkel exciton model for this aggregate ${ }^{18,19}$. The $816 \mathrm{~cm}^{-1}$ splitting is a measure of electronic coupling between chromophores.

Panels $\mathrm{Q}_{\mathrm{x} 1}$ and $\mathrm{Q}_{\mathrm{x} 2}$ show the next pair of modes. Compared to $\mathrm{Q}_{\mathrm{y}}$, the interaction is very weak and results in $\sim 170 \mathrm{~cm}^{-1}$ splitting. Contrary to $\mathrm{Q}_{\mathrm{y}}$, each $\mathrm{Q}_{\mathrm{x} 1}$ and $\mathrm{Q}_{\mathrm{x} 2}$ excited state wavefunction is heavily dominated by a single monomer because of the small interaction and the difference in $Q_{x}$ frequencies of $\alpha$ and $\beta$ monomers. Panels $\mathrm{B}_{\mathrm{x} 1}$ and $\mathrm{B}_{\mathrm{x} 2}$ display a pair of electronic modes corresponding to the $\mathrm{B}_{\mathrm{x}}$ state. Similar to $\mathrm{Q}_{\mathrm{y}}$ the strong transition dipoles of $\mathrm{B}_{\mathrm{x}}$ result in the large $\sim 734 \mathrm{~cm}^{-1}$ splitting, and these states are again near symmetric and antisymmetric combinations of the monomer excited state wavefunctions. Modes CT1 and CT2 are completely different from the Q and B dimer states. They delocalize over off-diagonal areas, reflecting the electronic coherence between chromophores, and have no intramonomer contributions (diagonal areas). This explains the very weak oscillator strengths of these transitions. Upon CT1 excitation, the hole becomes localized on $\alpha \mathrm{B} 850$ (x-axis), whereas the electron is transferred to $\beta \mathrm{B} 850$ (y-axis). On the other hand, state CT2 describes electron transfer from $\beta$ to $\alpha$. Thus CT1 and CT2 correspond to intermolecular charge-transfer excitations. These states lie just above the $\mathrm{Q}_{\mathrm{x}}$ transitions and form a band which overlaps with the $\mathrm{Q}_{\mathrm{x}}$ Frenkel exciton band in higher aggregates. Even though the CT modes are practically forbidden in linear absorption, they show up in nonlinear optical studies of these aggregates (e.g. electroabsorption).

These excitation frequencies of the monomer and dimer transitions provide all information necessary to construct the effective hamiltonian for this LH2 complex. The electronic coupling parameters $\mathrm{J}_{\mathrm{mn}}$ may be obtained by computing the excitation energies of the dimer built from the $\mathrm{m}$ and $\mathrm{n}$ chromophores by solving the eigenvalue problem ${ }^{11}$

$$
\left(\begin{array}{ll}
\Omega_{n} & J_{n m} \\
J_{m n} & \Omega_{m}
\end{array}\right)\left(\begin{array}{c}
\phi_{\alpha}(n) \\
\phi_{\alpha}(m)
\end{array}\right)=\varepsilon_{\alpha}\left(\begin{array}{l}
\phi_{\alpha}(n) \\
\phi_{\alpha}(m)
\end{array}\right)
$$

Here $\phi_{\alpha}(\mathrm{n})\left(\phi_{\alpha}(\mathrm{m})\right)$ and $\Omega_{\mathrm{n}}\left(\Omega_{\mathrm{m}}\right)$ are the monomeric excited state wavefunctions and their energies, $\varepsilon_{\alpha}$ are the energies of the dimer states. The electronic coupling may be computed from the solution of the Eq. (3)

$$
4 \mathrm{~J}_{\mathrm{mn}}^{2}=\Delta \varepsilon^{2}-\Delta \Omega^{2}
$$

where $\Delta \varepsilon=\varepsilon_{2}-\varepsilon_{1}$ is the electronic splitting between pair of dimer states and $\Delta \Omega=\Omega_{2}-\Omega_{1}$. The energies $\varepsilon_{2}\left(\varepsilon_{1}\right)$ and $\Omega_{2}\left(\Omega_{1}\right)$ are provided by a direct CEO calculation of the $(\mathrm{mn})$ dimer and each separate monomer. The contribution from other electronic states to the dimeric splitting is negligible when the states are well separated. Furthermore, a single CEO computation of a dimer gives the electronic couplings for all excited state pairs ${ }^{18}$.

By considering different dimers from the LH2 complex we computed the effective hamiltonians for the $\mathrm{Q}_{\mathrm{y}}$ and $B_{x}$ excitonic bands which dominate the linear response. The cartoons in Fig. 1 graphically display the computed electronic couplings among different chromophores of the Rs. molischianum LH2 complex. We first analyze intermolecular interactions between $\mathrm{Q}_{\mathrm{y}}$ states. As expected, closely spaced B850 molecules of the upper ring have a strong interaction. The intradimer coupling $\left(\sim 408 \mathrm{~cm}^{-1}\right)$ slightly exceeds its interdimer counterpart $\left(\sim 366 \mathrm{~cm}^{-1}\right)$. The interaction significantly decreases for next-nearest neighbors $\left(\sim 100 \mathrm{~cm}^{-1}\right)$, and further for third-nearest neighbors $\left(\sim 30 \mathrm{~cm}^{-1}\right)$. We found all other couplings to be smaller $10 \mathrm{~cm}^{-1}$. These results generally agree with couplings of the $\mathrm{Q}$ states computed using other approaches ${ }^{15,17,20}$. The upperlower ring interaction is much weaker. Each B800 pigment has significant coupling only to four nearest molecules from B850 ring. The computed B800-B850 electronic couplings $\left(\sim 50 \mathrm{~cm}^{-1}\right)$ are markedly larger than other calculations. Finally, the interaction between neighboring B800 pigments is small and practically vanishes for the next-to-nearest neighbor molecules. The $\mathrm{B}_{\mathrm{x}}$ electronic couplings shown in the lower graph of Fig. 1 are significantly smaller than their $\mathrm{Q}_{\mathrm{y}}$ counterparts. 
Figure 2. A: Structure of the $\alpha \beta B 850$ dimer. B: Calculated linear absorption spectrum of $\alpha \beta B 850$ dimer. Contour plots the electronic modes which dominate the optical absorption of B850 dimer. The axis labels represent the individual atoms. The atom labeling runs over $\beta$ molecule first. The panels indicate the electronic mode according to panel $\mathrm{B}$.
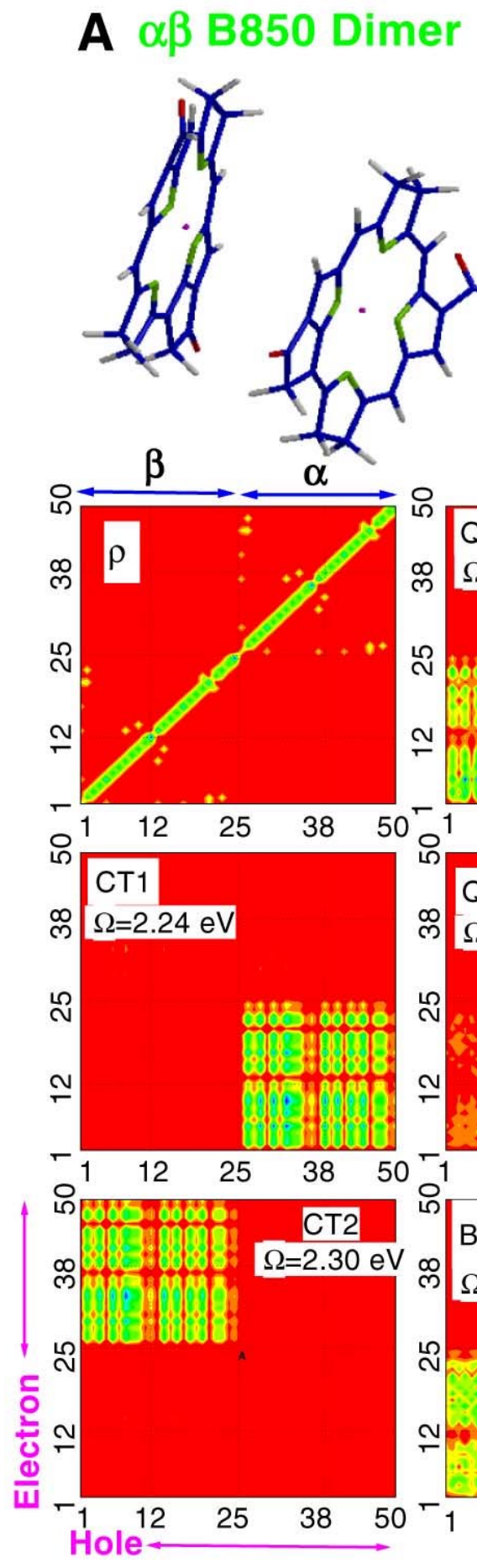

$\infty$

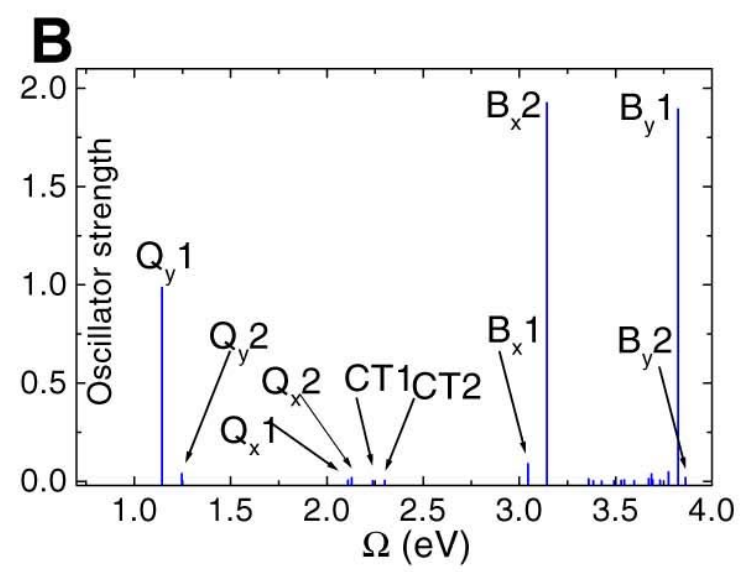

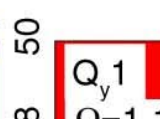
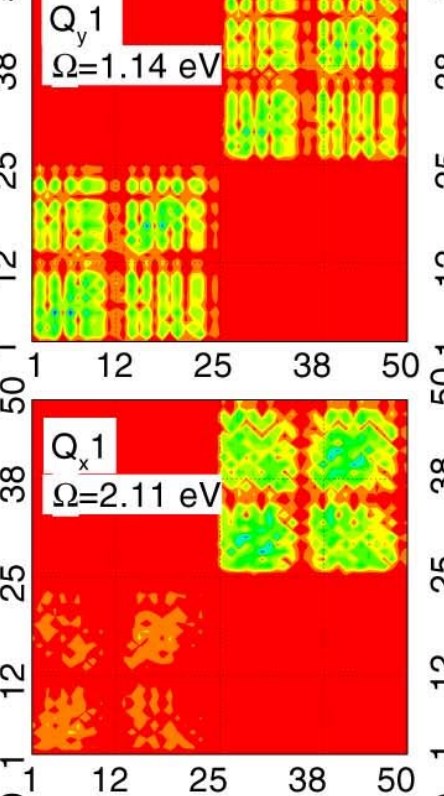

우
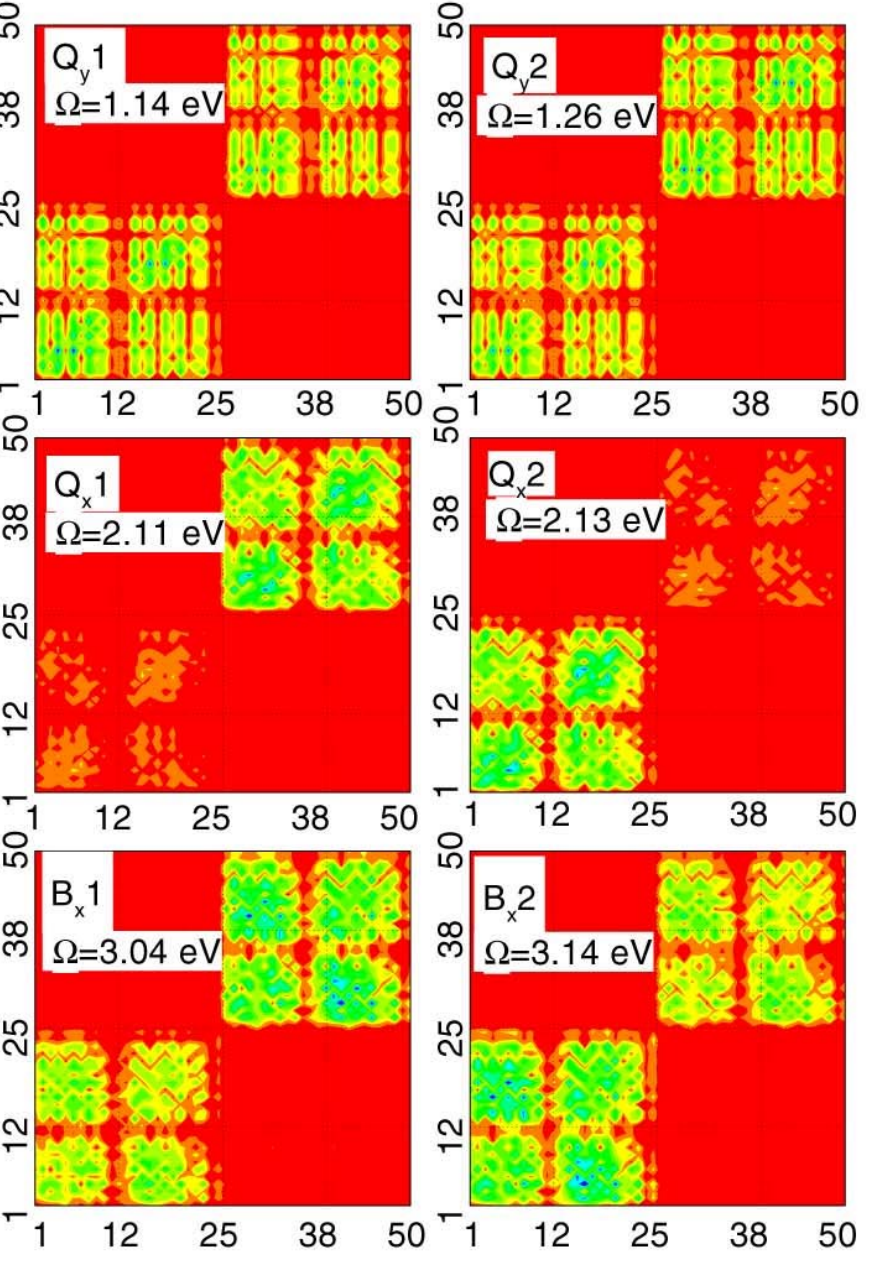


\section{Localized Electronic Excitations In Phenylacetylene Dendrimers.}

In this section we discuss a new class of interesting systems (phenylacetylene dendrimers) and show that they too can be described as coupled chromophores. Dendrimeric molecules with branched tree-like structures are drawing considerable attention ${ }^{2,21,22}$ (Fig. 3). The dynamics of photophysical (electronic and vibrational energy transfer) as well as photochemical processes has been demonstrated to be strongly affected by geometric confinement. Theoretical interest in these "Cayley trees" (also known as Bethe lattices) arises from their peculiar dimensionality: The connectivity between different sites is onedimensional (there is only one path to go between two points). However, the number of atoms grows exponentially with generation, as in infinite-dimensional systems. This leads to unusual transport and optical properties. Calculating the optical electronic excitations in these systems is a formidable task, and no simple method exists for analyzing the nature of these excitations and predicting their scaling with molecular size.

Figure 3. The generations (shown by different colors) in the extended dendrimers have a varying linear segment length. Their absorption frequency is blue-shifted for higher generations.

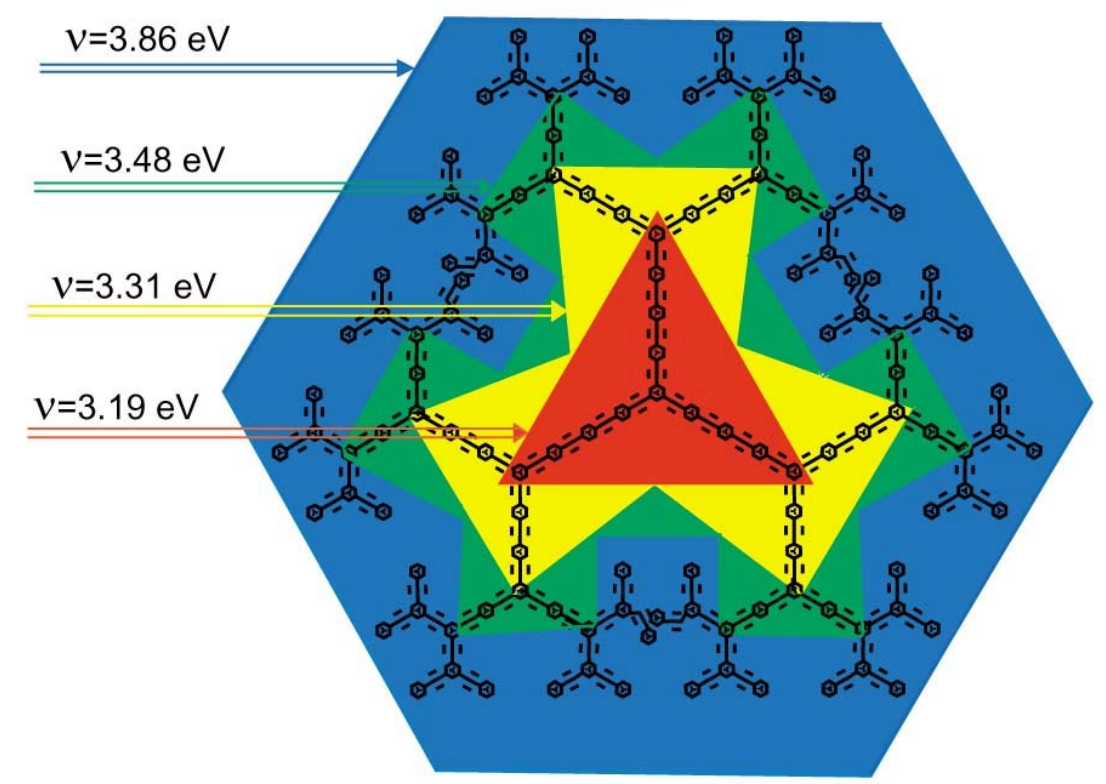

We have applied the CEO approach for calculating the absorption spectra of two families of phenylacetylene dendrimers (Fig.4 A and B) made out of phenylacetylene oligomer segments connected through para- or meta- substitutions of the benzene rings, leading to linear or zigzag chains respectively. These macromolecules have been suggested as artificial photonic antenna ${ }^{2,21,22}$. The absorption spectra of family A which has the same segment (linear unit) length in the various generations are essentially unchanged with molecular size. Family B (Fig. 4) has a varying segment length that decreases for higher generations. Here the absorption spectra (dashed lines in Fig. 4D) show new red-shifted features as the molecular size is increased. The following analysis shows how these trends arise naturally from the localized electronic excitations in these systems. It is difficult to anticipate this localization by inspecting the molecular orbitals, since the system is conjugated and the orbitals are delocalized. Nevertheless, the electron-hole pairs which contribute to the elementary optical collective excitations are well localized.

We have examined the linear (para- substituted) molecules (P-series) with $n=1,2,3$ repeat units (triple bonds) and the M7 molecule which consists of linear P1, P2, P3 segments connected at the meta- position with overall $n=7$ repeat units and represents a branch of the dendrimeters (see Fig.4C). The calculated linear absorption spectra of the P-oligomers have a single low-lying absorption peak (the band-edge transition (a)) which is significantly red-shifted with increasing chain length. The spectrum of M7 is markedly different; The single (a) line is split into three low-frequency transitions (a1, a2, and a3) which have the same frequencies as the band-edge transitions of its building linear blocks; the P1, P2, and P3 oligomers. 
Figure 4. The compact dendrimers (A) are made of the same linear building block P1. The extended dendrimers (B) have a varying linear segment length which decreases for higher generations; C: Structures and atom labeling of the linear para-oligomers Pn with $\mathrm{n}=1,2,3$ repeat units (triple bonds), and the M7 oligomer made of the P1, P2, P3 units conjugated at meta- position; D: Calculated (solid lines) and experimental (dashed lines) absorption spectra of the dendrimers; Contour plots of the electronic modes which dominate the absorption spectra of the oligomers shown in $\mathrm{C}$. The axes represent the carbon atoms. The spectrum of $\mathrm{M} 7$ is a sum of $\mathrm{P} 1+\mathrm{P} 2+\mathrm{P} 3$.

A

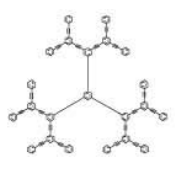

\section{D-22}

B

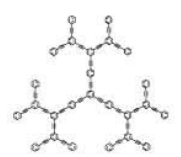

\section{D-25}

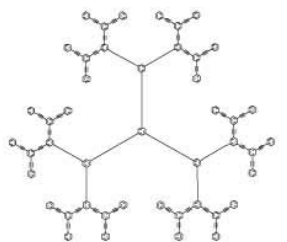

D-46

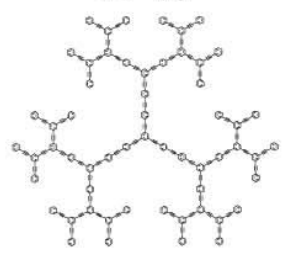

D-58

C
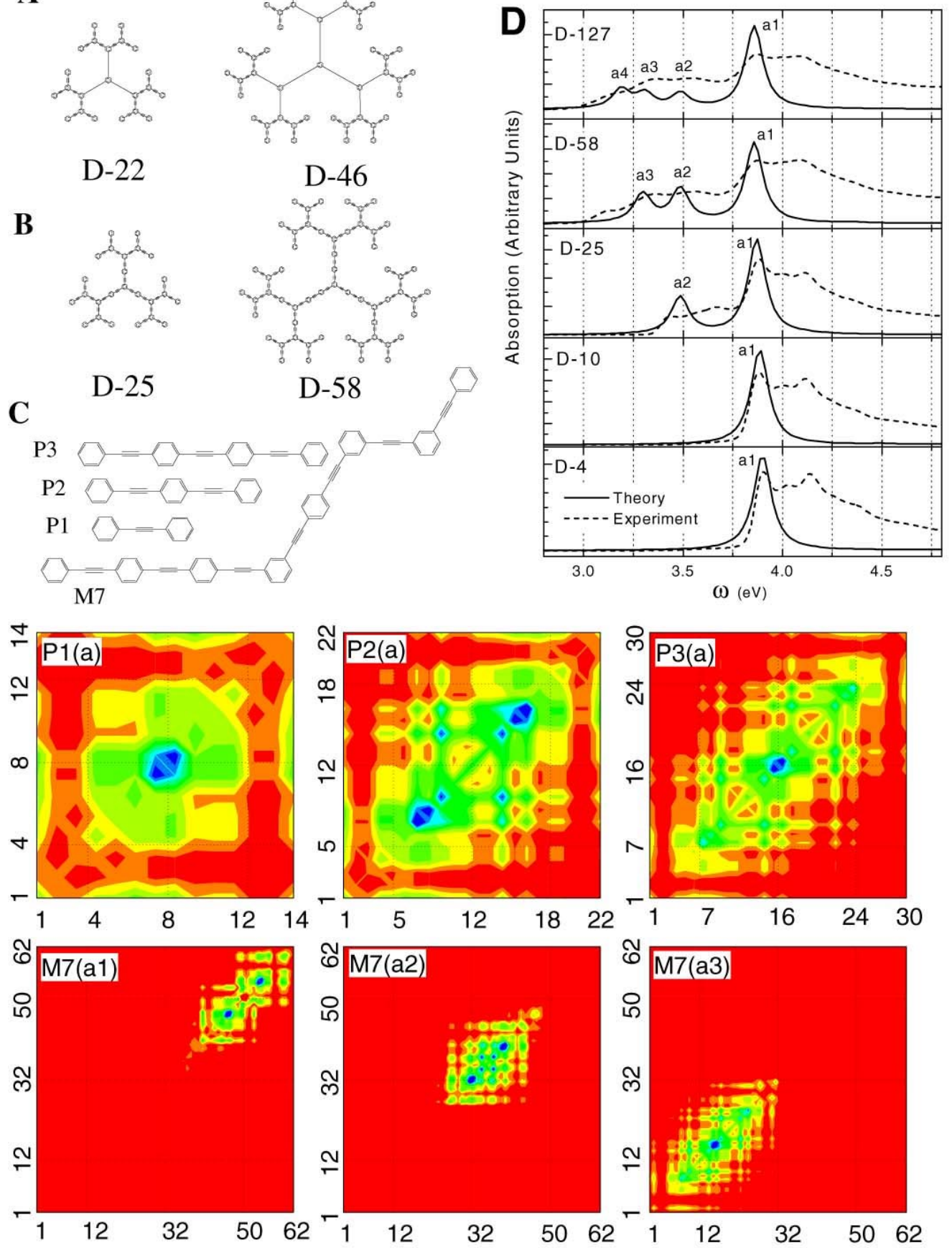
The collective modes corresponding to these electronic excitations are displayed in Fig.4. The top row of color plots shows the lowest frequency mode (a) of the para- oligomers. P1(a) centered at the triple bond shows maximum coherences and is delocalized over the entire molecule. The mode clearly shows that meta carbons $2,3,12$, and 13 have a vanishing electronic coherences with other carbon atoms. This is shown by the 'ring' around the plot with small coherences. Analogous patterns can be seen in mode (a) of longer linear oligomers $\mathrm{P} 2$, and $\mathrm{P} 3$. The boundary meta-atoms have vanishing coherences in all P-oligomers. The bottom row of color plots in Fig.4 displays the electronic modes of M7. Mode (a3) is localized at the P3 linear segment of M7 and is virtually identical to mode P3 (a). Similarly M7(a2) and M7(a1) resemble P2(a) and P1(a) respectively. The complete absence of coherence across meta- substitutions shown in this figure is remarkable; the optical excitations are clearly confined to the various segments. meta- conjugation makes a clear barrier for excitonic motion whereas para- conjugation is transparent to electronic coherences. This difference does not show up in the ground state, which is very similar for P7 and M7.

It is well established that meta-substituents are much less effective in changing reaction rates compared with their para-counterparts. This can be understood using resonant structures commonly used in organic chemistry which show that charges injected into the system by an nuclophilic or an electrophilic substituent are delocalized only at the ortho- and para- positions. The present study which establishes the same trend for electron-hole pairs created by light provides a direct link between spectroscopy and well-established rules-of-thumb for chemical reactivity. The lack of electronic coherence across meta- substitutions suggests that we can describe the optical excitations of dendrimers by dividing them into weakly-interacting chromophores. In zero-order we can neglect the interactions among chromophores altogether; The metaconjugated dendrimer behaves as a collection of its linear para-conjugated segments which interact with light independently. We modeled the absorption spectra of family A as a collection of P1 chromophores. The spectra thus only show one low frequency peak. The experimental and the modeled spectra of D-4 and D-10 members of family A are displayed in Fig.4D. The spectra of other generations are very similar ${ }^{22}$. We have similarly calculated the absorption spectra of the B family by simply adding the spectra of its segments. The resulting calculated and experimental spectra displayed in Fig.4 show that this simple procedure can produce the band edge red-shift trend as well as the relative peak intensities in these macromolecules, in complete agreement with experiment. Finally we estimated the interchromophore coupling in the compact dendrimers to be $\sim 70 \mathrm{~cm}^{-1}$ by applying approach described in the previous section. This coupling and the monomer energy are the parameters of Frenkel excition hamiltonian which was further applied to study linear and nonlinear response of the whole supermolecule ${ }^{23}$.

\section{Discussion}

In summary, we have demonstrated that the CEO approach offers a fast and inexpensive calculation of the Frenkel exciton hamiltonian in extended molecular aggregates and supermolecular structures without invoking the $\mathrm{PDA}^{14}$ or similar approximations as well as a real-space visualization of the various electronic excitations. The latter allows us to develop a natural framework for the interpretation electronic excitations in these systems. Instead of asking which of the many-electron states are most relevant, we can explore how do different regions of the molecule couple and affect each other. The ability to describe large conjugated systems as weakly coupled chromophores established here for LH2 antenna and phenylacetylene dendrimers opens up the possibility of applying an effective Frenkel exciton picture towards the theoretical modeling of their optical response ${ }^{19,23}$.

Acknowledgement. We wish to thank Prof. K. Schulten and Prof. M. Zerner for providing us with crystal structures of Rs. molischianum. We thank to Dr. S. F. Swallen and Prof. R. Kopelman for providing us with the linear absorption data and for most valuable discussions. The support of the National Science Foundation through Grant No. CHE-9526125 and the United States Air Force Office of Scientific Research (Grant No. F49620-96-1-0030), is gratefully acknowledged. 


\section{References}

(1) R. Lecuiller, J. Berrehar, C. Lapersonne-Meyer, M. Schott, Phys. Rev. Lett. 1998, 80, 4068; B. Kraabel, D. Hulin, C. Aslangul, C. Lapersonne-Meyer, M. Schott, J. Chem. Phys. 1998, 83, 227.

(2) D.-L. Jiang, T. Aida, Nature 1997, 388, 453; V. Balzani, S. Campagna, G. Denti, A. Juris, S. Serroni, M. Venturi, Acc. Chem. Res. 1998, 31, 26; J. Freechet, Science 1994, 263, 1710.

(3) F. Diederich, C. Thilgen, Science 1994, 271, 317; C. Boudon, et al. J. Am. Chem. Soc. 1998, $120,7860$.

(4) R. J. Pieters, F. Diederich, Chem. Commun. 1996, 19, 2255; R.J. Pieters, J. Cuntze, M. Bonnet, F. Diederich, J. Chem. Soc. Perk. T. 2 1997, 10, 1891.

(5) S.R. Forrest, Chem. Rev. 1997, 97, 1793.

(6) H. Zuber, R. A. Brunisholz, Photosynthesis: Physical Mechanism and Chemical Patterns, (Cambridge University Press: New York), 1980.

(7) J. Koepke, X. Hu, C. Muenke, K. Schulten, H. Michel, Structure 1996, 4, 581.

(8) J. Michl and V. Bonacic-Koutecky, Electronic Aspects of Organic Photochemistry, (Willey, New York), 1990; M. Klessinger and J. Michl, Excited States and Photochemistry of Organic Molecules (VCH, New York), 1995.

(9) S. Speiser, Chem. Rev. 1996, 96, 1953.

(10) A. S. Davydov, Theory of Molecular Excitons, (Plenum, NewYork, 1971).

(11) M. Kasha, H. R. Rawls, M. Ashraf El-Bayoumi, Pure and Applied Chem., 1965, 11, 371.

(12) S. Tretiak, V. Chernyak and S. Mukamel, J. Am. Chem. Soc., 1997, 119, 11408.

(13) S. Mukamel, S. Tretiak, T. Wagersreiter, V. Chernyak, Science, 1997, 277, 781.

(14) T. Pullerits, V. Sundstrom, Acc. Chem. Res. 1996, 29, 381.

(15) T. Pullerits, V. Sundstrom, R. van Grondelle, J.Phys.Chem. 1999, 103, 2327.

(16) B. P. Krueger, G. D. Scholes, G. R. Fleming, J. Phys. Chem. B, 1998, 102, 5378.

(17) G. D. Scholes, I. R. Gould, R. J. Cogdell, G. R. Fleming,, J. Phys. Chem. B, 1999,103, 2543.

(18) S. Tretiak, V. Chernyak and S. Mukamel, (unpublished).

(19) E. Poliakov, V. Chernyak, S. Tretiak, and S. Mukamel, J. Chem. Phys., 1999, 110, 8161.

(20) K. Sauer, R. J. Cogdell, S. M. Prince, A. A. Freer, N. W. Isaacs, H. Scheer, Photochem. Photobiol., 1996, 64, 564.

(21) S. Tretiak, V. Chernyak, and S. Mukamel, J. Phys. Chem. B 1998, 102, 3310.

(22) R. Kopelman, et al., Phys. Rev.Lett, 1997, 78, 1239; A. Bar-Haim, Y. Klafter, R. Kopelman, J. Am. Chem. Soc. 1997, 119, 6197.

(23) T. Meier, V.Chernyak and S.Mukamel, J.Phys.Chem.B, 1997,101, 7332. 